\title{
Supercritical Fluid Chromatographic Separation of Organophosphorus Pesticides on Packed Columns and Capillary Columns
}

\author{
Yasuhiko Nishikawa \\ Sumitomo Chemical Co., Ltd., Environmental Health Science Laboratory, \\ Takatsukasa, Takarazuka, Hyogo 665, Japan
}

\begin{abstract}
The separation of organophosphorus pesticides by supercritical fluid chromatography was investigated using four packed columns and five fused silica capillary columns. Fourteen pesticides used worldwide were selected; the separation of as many species as possible with one injection was attempted by optimizing the column temperature and the pressure program. Among the packed columns used, an ODS column resulted in a favorable separation, enabling the simultaneous identification of 12 species. However, pesticides having strong hydrogen-bonding functional groups were either not eluted or eluted later. By using capillary columns the chromatographic peaks of these pesticides could be obtained, and various separation modes were possible with liquid stationary phases having different polarities. Also presented is information concerning the retention behavior of each organophosphorus pesticide.
\end{abstract}

Keywords Organophosphorus pesticide, supercritical fluid chromatography, simultaneous separation, packed column, fused silica capillary column, liquid stationary phase

Organophosphorus compounds have been used worldwide as insecticides, fungicides, and herbicides, owing to their high insecticidal activity, wide spectra of action on plant pests, and characteristic of breaking down to form products that are nontoxic to man and animals. Even though one disadvantage is their relatively high toxicity to vertebrates, a large number of organophosphorus pesticides with moderate or low toxicity for mammals have recently been synthesized. One of these, fenitrothion, $O, O$-dimethyl $O$-4-nitro- $m$ tolyl phosphorothioate, has been registered in over 80 countries owing to its wide insecticidal spectrum coupled with favorable residue figures, as well as its relatively low mammalian toxicity.

Some chromatographers ${ }^{1-8}$ have reported the use of supercritical fluid chromatography (SFC) for various pesticide compounds. Most of these applications concerned carbamates. Because SFC requires no heated injection port or column oven, it offers the advantage of avoiding the thermal decomposition which can occur with gas chromatography (GC) in the case of trace analysis. Although organophosphorus pesticides have usually been analyzed by GC with selective detectors, such as FPD or FTD, a separation method using a lower temperature or a unique mobile phase with strong solvating power is desirable, due to the thermal lability or non-volatility of some compounds. ${ }^{9,10}$ This thermal isomerization problem becomes marked when analyzing technical material or formulation. Thus, supercritical fluid separation is a promising technique for detecting thermally labile pesticides or those of low volatility. An additional advantage of using SFC for the analysis of pesticides is the ease of coupling with supercritical fluid extraction (SFE), which is effective for sample pretreatment in residue analysis. ${ }^{11,12}$ However, few studies ${ }^{5,7,8}$ have been carried out concerning the separation of organophosphorus pesticides by SFC. Further, not many pesticides have been simultaneously determined and not many stationary phases have been investigated.

Multiresidue analytical methods are useful for identifying and determining more than one residue in food samples. Recent pesticide residue analysis often requires the use of such methodologies. Therefore, the need for a simultaneous determination of pesticides with analogous structures has recently been raised. Furthermore, various stationary phases are needed as a mixed-phase column for an efficient multiresidue determination of organophosphorus pesticides. ${ }^{13}$

In this study, the separation of 14 widely-used organophosphorus pesticides from low-toxicity pesticides, such as fenitrothion, to highly toxic ones, such as parathion, were examined by SFC using various stationary phases.

\section{Experimental}

\section{Reagents and materials}

The organophosphorus pesticides used in this study were prepared by Sumitomo Chemical Co., Ltd. (Osaka, 
Japan) or Wako Pure Chemical Co. (Osaka, Japan): butamifos [O-ethyl $O$-5-methyl-2-nitrophenyl $s$-butylphosphoramidothioate], cyanophos [O-4-cyanophenyl $O, O$-dimethyl phosphorothioate], diazinon [ $O, O$-diethyl O-2-isopropyl-6-methylpyrimidin-4-yl phosphorothioate], dichlorvos [2,2-dichlorovinyl dimethyl phosphate], dimethoate [O,O-dimethyl $S$-methylcarbamoylmethyl phosphorodithioate], EPN [O-ethyl $O$-4-nitrophenyl phenylphosphorothioate], fenitrothion [O,O-dimethyl $O$-4-nitro- $m$-tolyl phosphorothioate], fenthion [ $O, O$ dimethyl 0 -4-methylthio-m-tolyl phosphorothioate], malathion [diethyl(dimethoxythiophosphorylthio)succinate], methylparathion [O,O-dimethyl $O$-4-nitrophen-

Table 1 Structures of organophosphorus pesticides

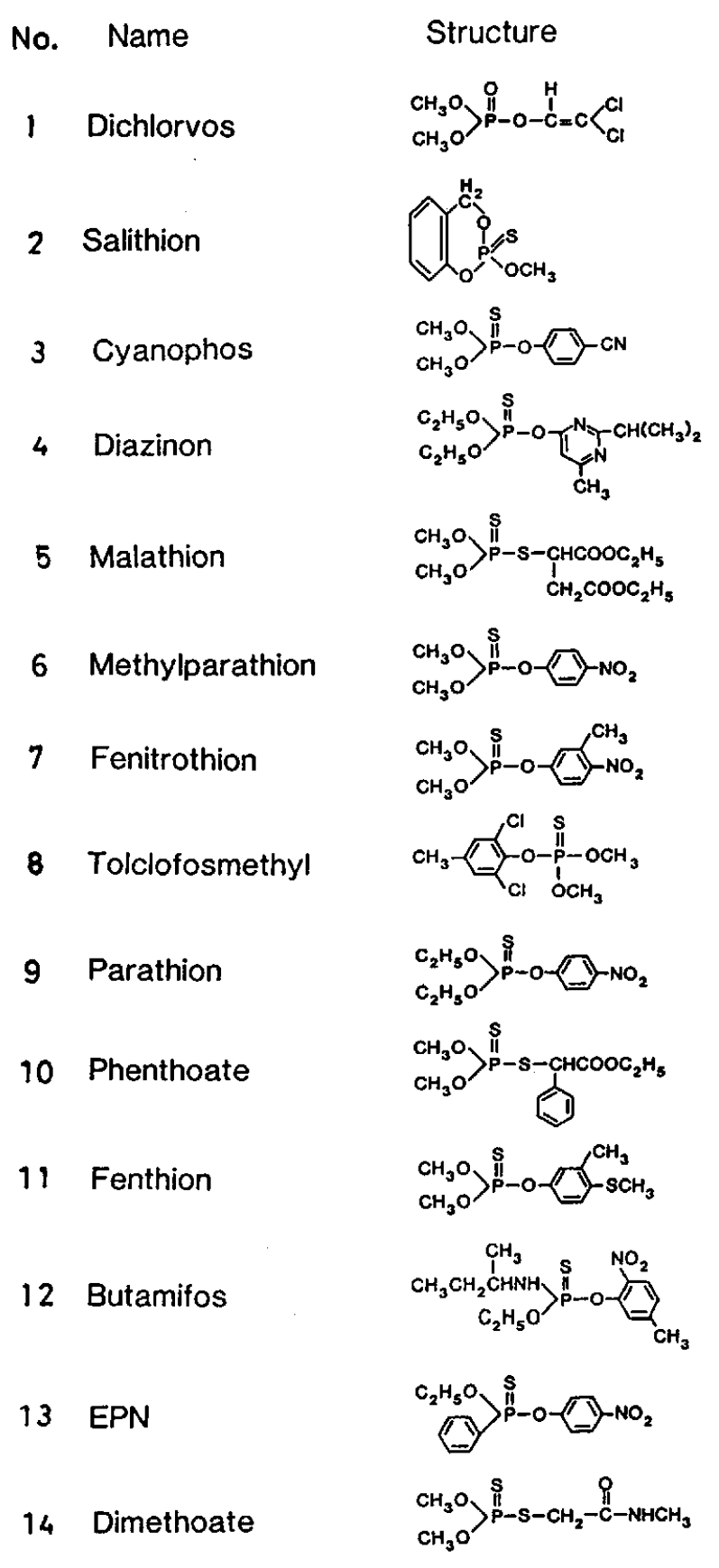

yl phosphorothioate], parathion [O,O-diethyl $O$-4nitrophenyl phosphorothioate], phenthoate [ethyl 2dimethoxythiophosphorylthio-2-phenylacetate], salithion [2-methoxy-4H-benzo-1,3,2-dioxaphosphorin 2sulfide], tolclofosmethyl [O-2,6-dichloro-4-methylphenyl $O, O$-dimethyl phosphorothioate]. Table 1 shows their structures. The acetone used for the preparation of each solution was of residue analytical grade (Kanto Chemical Co., Inc., Tokyo, Japan).

The packed columns used in this work were a Develosil $\mathrm{CN}-5$, a Develosil N.P.SILICA-5, a Develosil 60-5 $(0.5 \mathrm{~mm}$ i.d. $\times 200 \mathrm{~mm}, 5 \mu \mathrm{m})$ and a Develosil ODS-5 $(1.0 \mathrm{~mm}$ i.d. $\times 150 \mathrm{~mm}, 5 \mu \mathrm{m})$; these were supplied by Nomura Chemical Co. (Seto, Japan). Fused silica capillary SFC columns were purchased from $\mathbf{J} \&$ W Scientific, Inc. (Rancho Cordova, CA, USA): a DB-1, a DB-5, a DB-1701, a DB-225 and a DB-WAX, in order of increasing polarity. These column stationary phases comprised $100 \%$ dimethyl-polysiloxane (DB-1), 5\% diphenyl-95\% dimethyl-polysiloxane (DB-5), 14\% cyanopropyl phenyl-86\% dimethyl-polysiloxane (DB1701), 50\% cyanopropyl methyl-50\% phenyl methylpolysiloxane (DB-225) and polyethylene glycol $20 \mathrm{M}$ (DB-WAX), respectively. Their sizes were $0.1 \mathrm{~mm}$ i.d.X $20 \mathrm{~m}$, with a film thickness of $0.1 \mu \mathrm{m}$, except for DB-5 $(0.05 \mathrm{~mm}$ i.d. $\times 10 \mathrm{~m})$.

The purity of the carbon dioxide (Neriki Gas Co., Amagasaki, Japan) used as the mobile phase was $99.5 \%$.

\section{Apparatus}

The SFC system comprised a Brownlee Micro Gradient System (Applied Biosystems Inc., Foster City, CA, USA), which is a pump capable of pressure programming, and a Shimadzu GC-9A (Kyoto, Japan) equipped with a FID and a Valco A90 6-way valve injector (Houston, TX, USA). The pump, whose cylinder was pressurized with liquid carbon dioxide without cooling of the pump head, was used for fluid delivery. While the GC oven was maintained at over the critical temperature, the injection valve was kept at room temperature. For packed columns, all of the samples were introduced into the column at an injection volume of $0.2 \mu \mathrm{l}$. For capillary columns, samples introduced at an injection volume of $0.06 \mu \mathrm{l}$ were split via a splitter before reaching the separation column. A tapered restrictor was connected to the column outlet through a joint without any dead volume. The restrictor was prepared by heating and pulling the end of $50 \mu \mathrm{m}$ i.d. fused silica capillary tubing (GL Science Inc., Tokyo, Japan). The restrictor end was designed according to the optimum flow rate for the column type with reference to the work of Schwartz et al. ${ }^{14}$ Chromatograms were obtained with a Shimadzu integrator C-R3A.

\section{Results and Discussion}

In this work the control of the column temperature or the pressure program was studied in order to 
simultaneously separate as many of the 14 pesticides studied as possible. Figures $1-4$ show chromatograms of the organophosphorus pesticide mixtures obtained using ODS, cyano, porous silica and non-porous silica packed columns. The column temperature and pressure were optimized in order to obtain the most

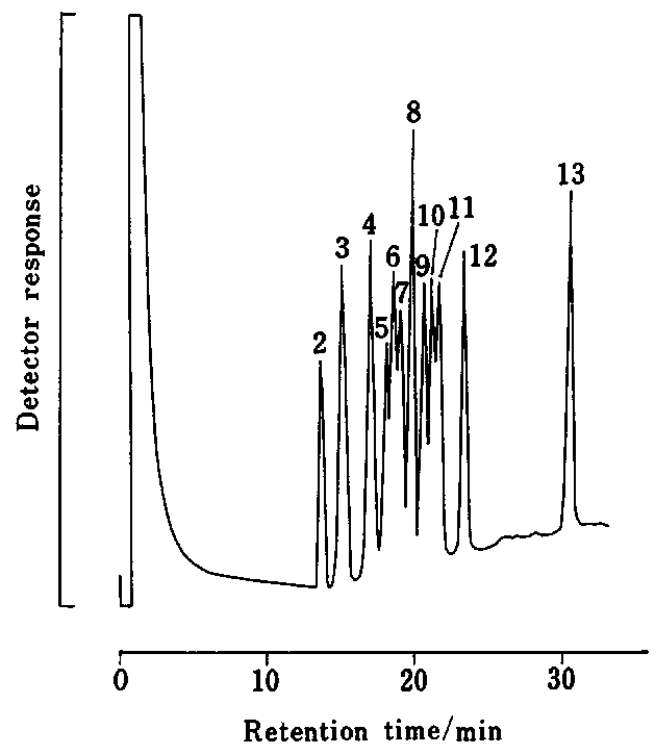

Fig. 1 SFC chromatogram of organophosphorus pesticides with an ODS column. Column: Develosil ODS-5. Conditions: pressure programmed from $1500 \mathrm{psi}(1 \mathrm{psi}=6.895 \times$ $10^{3} \mathrm{~Pa}$ ) to $2700 \mathrm{psi}$ at $40 \mathrm{psi} / \mathrm{min}$ after a 2 -min isobaric period; temperature held at $120^{\circ} \mathrm{C}$. The numbers of peaks correspond to the numbers given in Table 1.

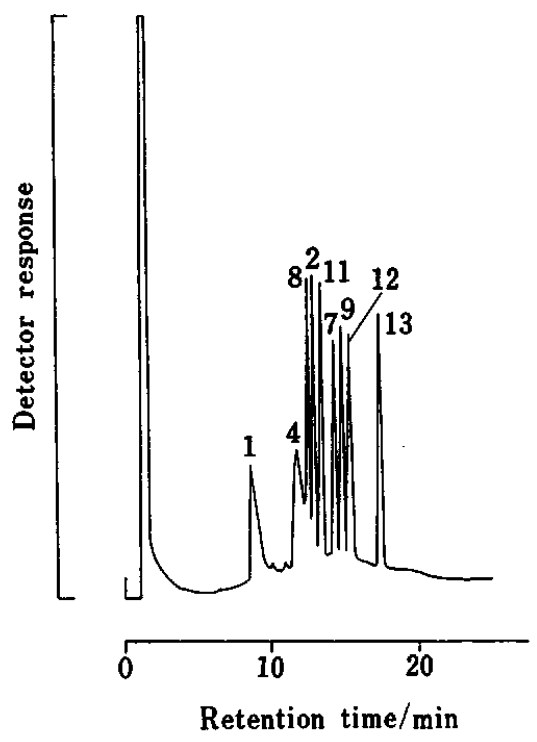

Fig. 2 SFC chromatogram of organophosphorus pesticides with a cyano column. Column: Develosil CN-5. Conditions: pressure programmed from $1500 \mathrm{psi}$ to $3800 \mathrm{psi}$ at $100 \mathrm{psi} / \mathrm{min}$ after a 2 -min isobaric period; temperature held at $80^{\circ} \mathrm{C}$. The numbers of peaks correspond to the numbers given in Table 1 . favorable chromatograms for simultaneous separation. As shown in Fig. 1, by using an ODS column, the 12 organophosphorus pesticides tested, except for dichlorvos and dimethoate, could be separated in one injection. By using a cyano column (Fig. 2), nine pesticides were separated. A non-porous silica column

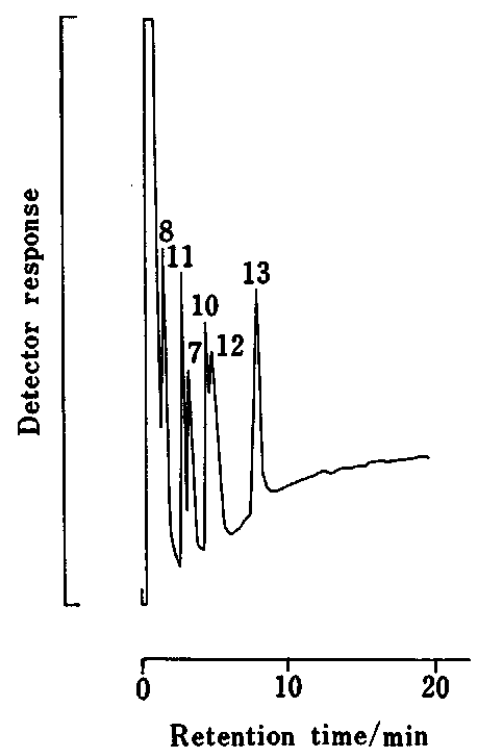

Fig. 3 SFC chromatogram of organophosphorus pesticides with a non-porous silica column. Column: Develosil N.P.SILICA-5. Conditions: pressure programmed from $1500 \mathrm{psi}$ to $2500 \mathrm{psi}$ at $50 \mathrm{psi} / \mathrm{min}$ after a 2 -min isobaric period; temperature held at $100^{\circ} \mathrm{C}$. The numbers of peaks correspond to the numbers given in Table 1 .

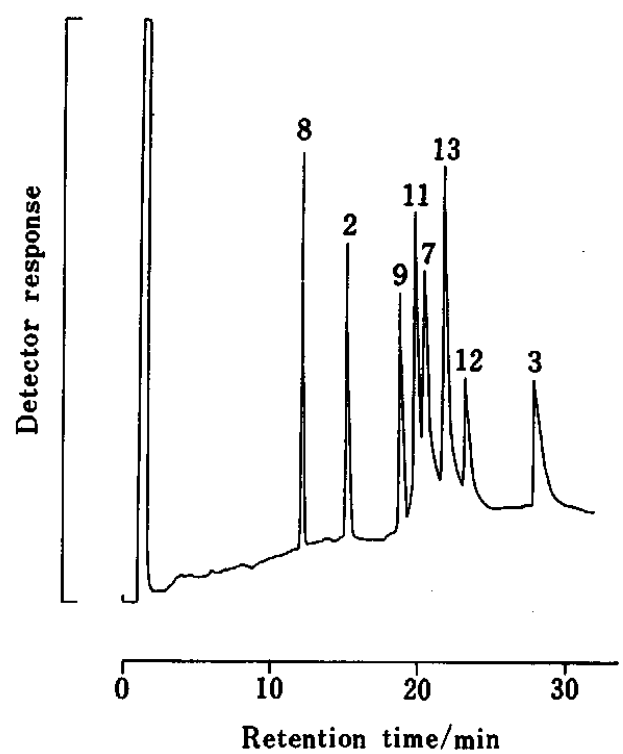

Fig. 4 SFC chromatogram of organophosphorus pesticides with a porous silica column. Column: Develosil 60-5. Conditions: pressure programmed from $1500 \mathrm{psi}$ to $4500 \mathrm{psi}$ at $100 \mathrm{psi} / \mathrm{min}$ after a 2 -min isobaric period; temperature held at $50^{\circ} \mathrm{C}$. The numbers of peaks correspond to the numbers given in Table 1 . 
has the advantage of being capable of eluting diazinon (4), dichlorvos (1) and malathion (5), which could not be eluted with the porous silica column (cf. Table 2). Six pesticides could be separated in one injection using the non-porous silica column (Fig. 3) and eight with the porous silica column (Fig. 4).

Dimethoate (14) could not be eluted with any of the columns examined. The reason is thought to be that dimethoate has a functional group that forms a strong hydrogen bond which causes a significant interaction between the functional group and a residual silanol group on the surface of packing materials ( $c f$. Fig. 5). The adsorption behavior of diazinon (4), dichlorvos (1) and malathion (5) on the porous silica column can be also explained by interactions between the strong hydrogenbonding functional groups and the silanol groups (Fig. 5).

With the capillary columns, deactivation of the liquid phases is supposed to result in both elution of these pesticides and higher separation efficiency. Figures 6-8 show chromatograms of organophosphorus pesticide mixtures obtained using DB-1, DB-225 and DB-WAX columns. The column temperature and pressure conditions were also optimized for favorable separation.

Table 2 Elution order of organophosphorus pesticides in packed SFC and capillary SFC

\begin{tabular}{|c|c|c|c|c|c|c|c|c|c|}
\hline Chromatography & & Pack & SFC & & & & pillary $s$ & & \\
\hline Column & ODS & Cyano & N.P.Silica & Porous Silica & $D B-1$ & $D B-5$ & DB-1701 & DB-225 & DB-WAX \\
\hline Short & $\begin{array}{c}1 \\
2 \\
3 \\
4 \\
5 \\
6 \\
7 \\
8 \\
9 \\
10 \\
11 \\
12 \\
13\end{array}$ & $\begin{array}{c}1 \\
4 \\
8 \\
2 \\
11 \\
3 \\
5 \\
7 \\
10 \\
6 \\
9 \\
12 \\
13\end{array}$ & $\begin{array}{c}2 \\
8 \\
1 \\
3 \\
11 \\
9 \\
6 \\
7 \\
4 \\
10 \\
12 \\
5 \\
13\end{array}$ & $\begin{array}{c}8 \\
2 \\
9 \\
11 \\
6 \\
7 \\
13 \\
12 \\
3 \\
10\end{array}$ & $\begin{array}{c}1 \\
2 \\
4 \\
3 \\
14 \\
5 \\
8 \\
6 \\
7 \\
9 \\
11 \\
10 \\
12 \\
13\end{array}$ & $\begin{array}{c}1 \\
2 \\
4 \\
14 \\
3 \\
5 \\
6 \\
7 \\
8 \\
9 \\
11 \\
10 \\
12 \\
13\end{array}$ & $\begin{array}{c}1 \\
4 \\
2 \\
3 \\
8 \\
14 \\
5 \\
6 \\
11 \\
7 \\
9 \\
10 \\
12 \\
13\end{array}$ & $\begin{array}{c}1 \\
4 \\
5 \\
8 \\
2 \\
3 \\
11 \\
10 \\
9 \\
7 \\
6 \\
12 \\
14 \\
13\end{array}$ & $\begin{array}{c}1 \\
4 \\
5 \\
8 \\
9 \\
10 \\
12 \\
2 \\
3 \\
11 \\
7 \\
6 \\
14 \\
13\end{array}$ \\
\hline
\end{tabular}

The numbers of chemicals correspond to the numbers given in Table 1.

\section{Dimethoate}

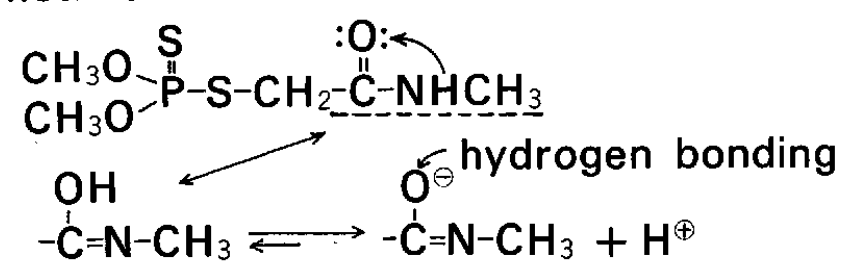

Diazinon

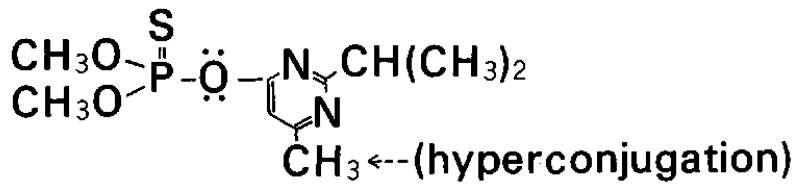

\section{Dichlorvos}

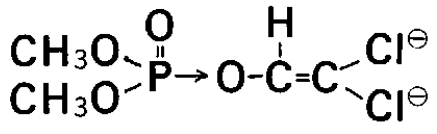

Malathion<smiles>COC(=O)CC(CC(=O)OC)C(=O)OP(O)(=S)OC</smiles>

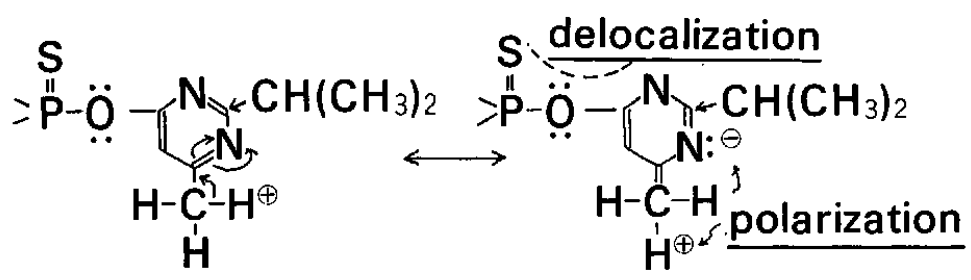

Fig. 5 Interaction between functional groups of the pesticides and the residual silanol groups on the surface of the packing. 
In all of the capillary columns examined, the elution of dimethoate and other pesticides, which could not be eluted with packed columns, was possible. Chromatographic peaks of all of the 14 pesticides tested were obtained with high separation efficiency. The use of capillary columns solved the problems of both solute abstraction (adsorption) and tailing, which were

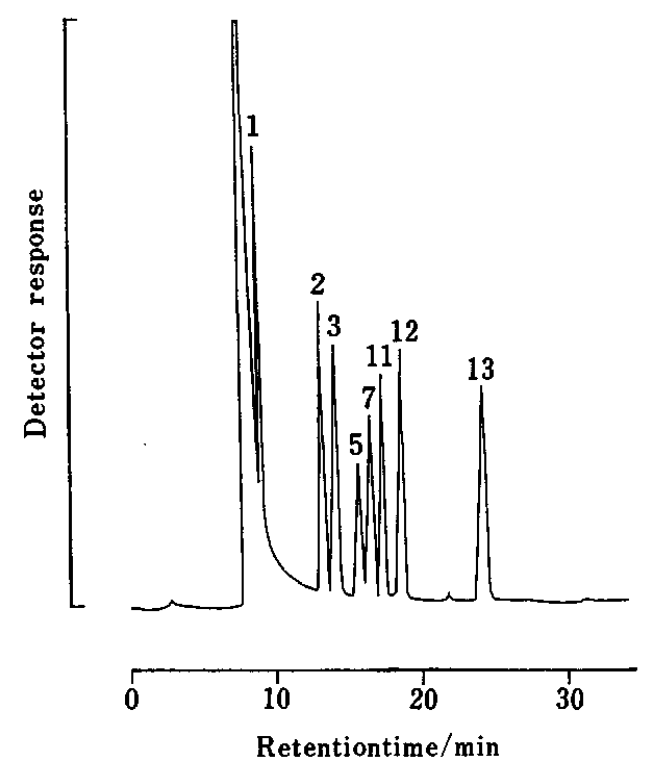

Fig. 6 SFC chromatogram of organophosphorus pesticides with a DB-1 column. Conditions: pressure programmed from $1600 \mathrm{psi}$ to $2700 \mathrm{psi}$ at $35 \mathrm{psi} / \mathrm{min}$ after a 2 -min isobaric period; temperature held at $95^{\circ} \mathrm{C}$. The numbers of peaks correspond to the numbers given in Table 1 .

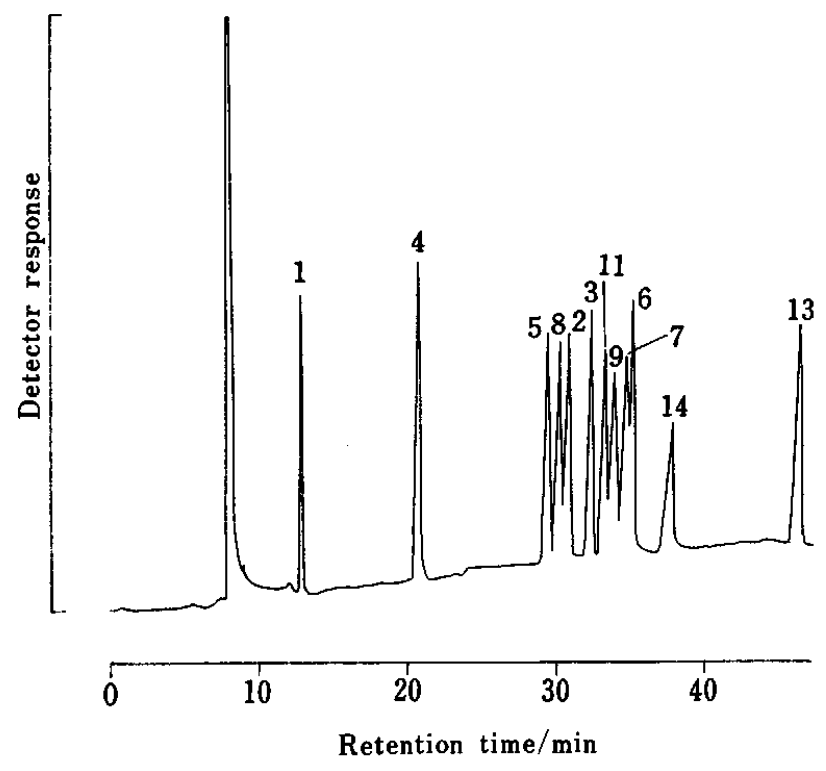

Fig. 7 SFC chromatogram of organophosphorus pesticides with a DB-225 column. Conditions: pressure programmed from $1500 \mathrm{psi}$ to $2900 \mathrm{psi}$ at $30 \mathrm{psi} / \mathrm{min}$ after a 2 -min isobaric period; temperature held at $90^{\circ} \mathrm{C}$. The numbers of peaks correspond to the numbers given in Table 1. especially marked with the porous silica column (Fig. 4). These results indicate that modifier solvents are needed to a lesser extent due to the introduction of fused silica capillary columns.

As can be seen from Figs. 6-8, more efficient separation is possible using a higher polarity liquid phase. With more polar capillary columns, like DB-225 or DB-WAX, more than 10 species can be separated with one injection, although the time needed for separation becomes slightly longer. In practice, a less polar liquid phase should be used for rapid separation. A more polar liquid phase should be used for retention-selective separation.

The chromatograms and the elution order of organophosphorus pesticides for the various stationary phases in Table 2 offer information concerning the retention behavior of organophosphorus compounds on stationary phases with different polarity. For example, a greater stationary phase polarity leads to an earlier elution of tolclofosmethyl (8) and a later elution of dimethoate (14). These results can be explained in terms of electronic considerations.

As a result of a resonance effect in tolclofosmethyl (Fig. 9 (a)), it is expected that a lower electron density of the oxygen atom whose electrons are drawn toward the phosphorus atom, leads to localized electrons in the sulfur atom. Tolclofosmethyl (8), although it eluted near the retention time of fenitrothion (7) with DB-1 or DB-5, is eluted earlier with DB-1701 or DB-225. This is attributed to a repulsion between the negative charges of the sulfur and the localized density of $\pi$-electron on the stationary phase, according to the extent of introduction

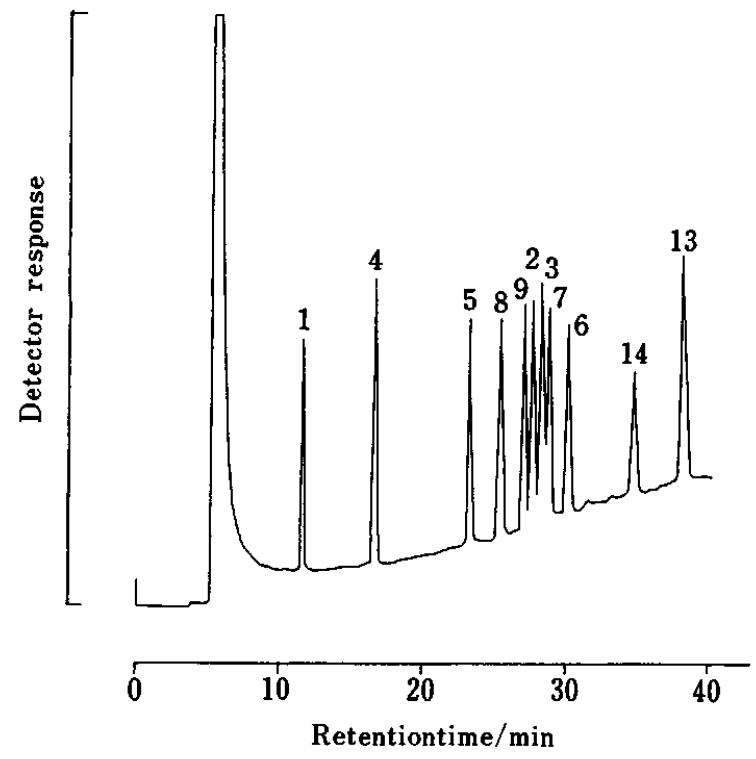

Fig. 8 SFC chromatogram of organophosphorus pesticides with a DB-WAX column. Conditions: pressure programmed from $1500 \mathrm{psi}$ to $3500 \mathrm{psi}$ at $50 \mathrm{psi} / \mathrm{min}$ after a $2-\mathrm{min}$ isobaric period; temperature held at $100^{\circ} \mathrm{C}$. The numbers of peaks correspond to the numbers given in Table 1. 
(a) Tolclofosmethyl<smiles>COP(=O)(OC)Oc1c(Cl)cc(C)cc1Cl</smiles>

(b) Dimethoate

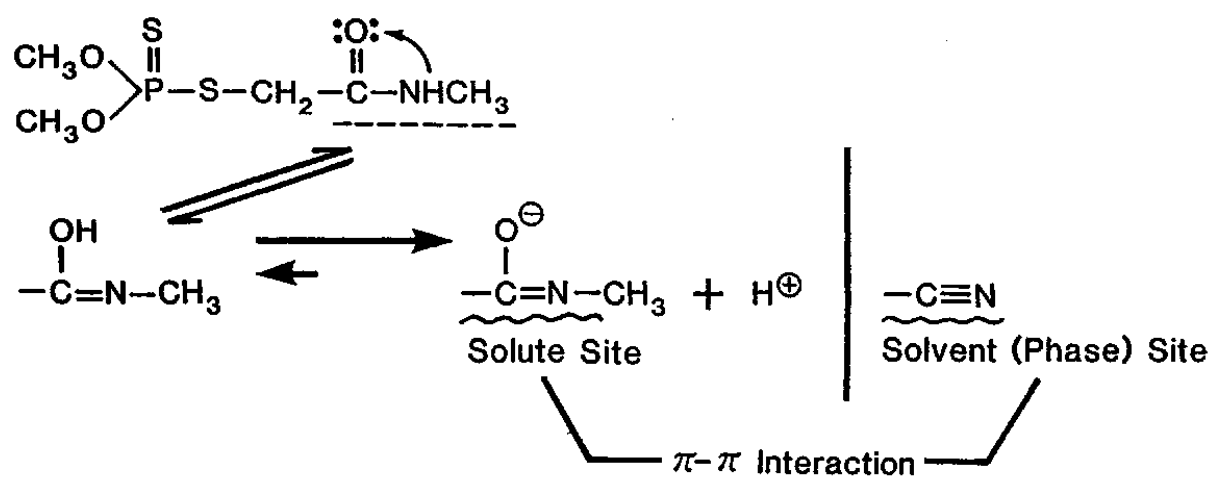

Fig. 9 Electronic consideration for tolclofosmethyl and dimethoate.

of the cyanopropyl group.

Figure 9 (b) shows the localized electronic structure expected for dimethoate (14). The solute (dimethoate) can contribute to $\pi$-electrons which are subject to hydrogen-bonding; the solvent (polar stationary phase DB-225) also has $\pi$-electrons in the triple bond $(-\mathrm{C} \equiv \mathrm{N})$. It can be assumed that $\pi-\pi$ interactions among them cause the delayed elution of dimethoate, compared with the case of DB-1 or DB-5.

A systematic explanation of the above-mentioned behavior would require the steric parameters related to the shape or size of each molecule, for example, the molar refraction or molar volume. Retention data under isobaric conditions should be a key to utilizing these parameters. A further investigation is under way.

\section{References}

1. B. W. Wright and R. D. Smith, J. High Resolut. Chromatogr. Chromatogr. Commun., 8, 8 (1985).

2. B. W. Wright and R. D. Smith, J. High Resolut. Chromatogr. Chromatogr. Commun., 9, 73 (1986).

3. B. W. Wright, H. T. Kalinoski, H. R. Udseth and R. D. Smith, J. High Resolut. Chromatogr. Chromatogr.
Commun., 9, 145 (1986).

4. A. J. Berry, D. E. Games and J. R. Perkins, J. Chromatogr., 363, 147 (1986).

5. K. E. Markides, E. D. Lee, R. Bolick and M. L. Lee, Anal. Chem., 58, 740 (1986).

6. H. T. Kalinoski, H. R. Udseth, B. W. Wright and R. D. Smith, J. Chromatogr., 400, 307 (1987).

7. H. T. Kalinoski and R. D. Smith, Anal. Chem., 60, 529 (1988).

8. W. T. Foreman, C. L. Shellum, J. W. Birks and R. E. Sievers, J. Chromatogr., 465, 23 (1989).

9. A. R. C. Hill, J. P. G. Wilkins, N. R. I. Findlay and K. E. M. Lontay, Analyst [London], 109, 483 (1984).

10. J. P. G. Wilkins, A. R. C. Hill and D. F. Lee, Analyst [London], 110, 1045 (1985).

11. M. E. McNally and J. R. Wheeler, J. Chromatogr., 435, 63 (1988).

12. Y. Nishikawa, Anal. Sci., 7, 567 (1991).

13. L. P. Van Dyk, I. H. Wiese and J. E. C. Mullen, in "Residue Reviews", Vol. 82, ed. F. A. Gunther, p. 67, Spring-Verlag, New York, Heidelberg, Berlin, 1982.

14. H. E. Schwartz, P. J. Barthel, S. E. Moring and H. H. Lauer, $L C-G C, 5,490$ (1987).

(Received April 11, 1992)

(Accepted July 13, 1992) 